\title{
Low-modulus PMMA bone cement modified with castor oil
}

\author{
Alejandro López, Andreas Hoess, Thomas Thersleff, Marjam Ott, Håkan Engqvist and \\ Cecilia Persson * \\ Department of Engineering Sciences, Division of Applied Materials Science, The Ångström \\ Laboratory, Uppsala University, Uppsala, Sweden
}

Received 11 August 2011

Accepted 17 February 2012

\begin{abstract}
Some of the current clinical and biomechanical data suggest that vertebroplasty causes the development of adjacent vertebral fractures shortly after augmentation. These findings have been attributed to high injection volumes as well as high Young's moduli of PMMA bone cements compared to that of the osteoporotic cancellous bone. The aim of this study was to evaluate the use of castor oil as a plasticizer for PMMA bone cements. The Young's modulus, yield strength, maximum polymerization temperature, doughing time, setting time and the complex viscosity curves during curing, were determined. The cytotoxicity of the materials extracts was assessed on cells of an osteoblast-like cell line. The addition of up to $12 \mathrm{wt} \%$ castor oil decreased yield strength from 88 to $15 \mathrm{MPa}$, Young's modulus from 1500 to $446 \mathrm{MPa}$ and maximum polymerization temperature from 41.3 to $25.6^{\circ} \mathrm{C}$, without affecting the setting time. However, castor oil seemed to interfere with the polymerization reaction, giving a negative effect on cell viability in a worst-case scenario.
\end{abstract}

Keywords: Bone cement, PMMA, low-modulus, castor oil, cytotoxicity

\section{Introduction}

Poly(methyl methacrylate) (PMMA) bone cements are widely used in clinics for vertebral augmentation procedures such as vertebroplasty (VP) and balloon kyphoplasty (BKP) [1]. Vertebral augmentation with PMMA, greatly increases the Young's modulus of the treated vertebra, as its modulus is much higher than the surrounding osteoporotic cancellous bone [2-4]. Some examples of commercial acrylic bone cements that are used for VP and their correspondent compressive moduli $(E)$ are: Simplex ${ }^{\circledR} \mathrm{P}$ (Stryker, Kalamazoo, MI, USA) $(E=1590 \mathrm{MPa} ; 2910 \mathrm{MPa})$ [5,6]; Osteopal ${ }^{\circledR}$ G (Heraeus GmbH, Hanau, Germany) ( $E=1740 \mathrm{MPa}$ ) [5]; and Vertecem (Synthes, Inc., West Chester, PA, USA) $(E=1837 \mathrm{MPa})$ [7]. The product KyphX ${ }^{\circledR}$ HV-R $^{\mathrm{TM}}$ (Kyphon Inc., Sunnyvale, CA, USA) $(E=3700$ $\mathrm{MPa}$ ) [6] is used for balloon kyphoplasty. An ideal bone cement should have a Young's modulus which is low enough to resemble that of the cancellous bone, while maintaining sufficient yield strength $\left(\sigma_{\mathrm{y}}\right)$ to resist the axial stresses supported by the spine. The axial $\sigma_{\mathrm{y}}$ has been reported to range between 2.5$15 \mathrm{MPa}$ and 0.1-8 MPa for normal and osteoporotic cancellous bone, respectively [8]. Additionally, the axial $E$ ranges between $100-500$ and $0.1-280 \mathrm{MPa}$, respectively [8].

\footnotetext{
*Address for correspondence: Cecilia Persson, The Ångström Laboratory, Lägerhyddsvägen 1, Box 534, 75121 Uppsala, Sweden. Tel.: +46 18471 7961; Fax: +46 18471 3572; E-mail: cecilia.persson@ angstrom.uu.se.
} 
During the past fifteen years, many researchers have related vertebroplasty using high-modulus acrylic bone cements, with the incidence of adjacent vertebral fractures. In 1997 Grados et al. [9] examined 40 patients that were treated with VP between 1990 and 1996. The results showed that there was a significantly increased probability of fracture in the area surrounding the augmented vertebra. Uppin et al. [10] evaluated 177 patients treated with VP after 2 years, and concluded that a considerable amount of patients with osteoporosis develop further fractures, and that two-thirds of these fractures take place in the adjacent vertebral bodies. Trout et al. [11] studied the risk and timing of fractures following VP in 432 patients and concluded that VP increases the chance of developing adjacent vertebral fractures, and those occur sooner than the non-adjacent ones. More recently, Chen et al. [12] proposed that cement leakage into the disk might be the cause of adjacent fractures based on a retrospective study on 138 patients that underwent VP. Biomechanical studies have been performed by Baroud et al. [13,14], where it was concluded that the maximum filling approach and an inward bulge of the endplate adjacent to the augmented vertebra might contribute to adjacent fractures. Berlemann et al. [15] analyzed 10 pairs of human cadaveric vertebrae, in which one vertebra of each pair was fully augmented with low-viscosity bone cement. The results gave lower failure strength for treated pairs compared to untreated ones; moreover, in each pair, the non-treated vertebra fractured. Wilcox et al. [16] performed finite element modeling of a lumbar L2-L3 spinal unit by simulating VP with different volumes of cement. The results indicated that there is an increased risk of adjacent fractures after VP and that it increases with the volume of injected cement.

Not all investigations support the conclusions that VP directly leads to an increased risk of adjacent fractures; Klazen et al. [17] e.g., report that the initial number of fractures is a more critical factor. The previously cited literature nevertheless provides a compelling reason for the development of low-modulus bone cements, leading to a significant amount of work in this direction. Boger et al. [18] compared the performance of cadaveric vertebral pairs treated with low-modulus and regular cement and concluded that, by using low-modulus cement, the failure strength of the adjacent segments was maintained closer to initial levels. They later introduced porosity into commercial PMMA cements, by incorporating a high viscosity solution of hydroxypropylmethyl cellulose, to decrease the Young's modulus in a controlled manner [19]. The results showed that $E$ could be decreased from $2800 \pm 70$ to $120 \pm$ $150 \mathrm{MPa}$ when increasing the amount of aqueous phase; however, the maximum compression strength $\left(\sigma_{\max }\right)$ also decreased from $170 \pm 5$ to $8 \pm 9 \mathrm{MPa}$ [19]. The use of these types of cements in orthopedics still remains to be assessed. Similar low-modulus cement was prepared by incorporating a sodium hyaluronate solution into commercial PMMA cement [7]. This formulation showed a reduction of $E$ from $1837 \pm 65$ to $47 \pm 26 \mathrm{MPa}, \sigma_{\max }$ from $91.0 \pm 5.3$ to $1.3 \pm 1.0 \mathrm{MPa}$, and maximum polymerization temperature ( $\left.T_{\max }\right)$ from $68 \pm 4^{\circ} \mathrm{C}$ to $35 \pm 1^{\circ} \mathrm{C}$, when increasing the amount of sodium hyaluronate solution [7]. However, it exhibited particle release that was much higher than in regular bone cement, which prevented it from further in vitro and in vivo studies [20]. Recently, Lam et al. [21] proposed a PMMA cement formulation containing strontium-substituted hydroxyapatite-nanoparticles with $15 \mathrm{vol} \%$ linoleic acid. This formulation exhibited a reduction of $E$ from $2142 \pm 129$ to $774 \pm 70 \mathrm{MPa}$ while maintaining relatively similar values of $\sigma_{\max }$, which varied from $63 \pm 2$ to $49 \pm 2 \mathrm{MPa}$ [21]. Nonetheless, negative cytotoxic effects were reported due to lower monomer conversion, and $\mathrm{N}, \mathrm{N}$-dimethyl- $p$-toluidine release from the materials containing linoleic acid [21].

Chemically modified (e.g., epoxidized) and unmodified vegetable oils are used as plasticizers for poly(vinyl chloride) [22], and natural rubber [23]. The selection of castor oil was based on that it was expected to lower the $T_{\mathrm{g}}$ of PMMA by accommodating within the polymer network, thereby reducing intermolecular interactions and cohesion [24]. Even though the study by Lam et al. [21] was not published at the time that castor oil was chosen for the present study, castor oil has a lower content of double 
bonds per mole than linoleic acid, which makes it less susceptible to radical attack than linoleic acid. The castor seed oil (castor oil) is a type of triglyceride extracted from the seeds of Ricinus communis. The Food and Drug Administration (FDA) and the Joint Food and Agriculture Organization (FAO)/World Health Organization (WHO), generally recognize castor oil as safe. It is commonly used in cosmetics and as a laxative [25]. Castor oil is also applied in polymer science as natural precursors for the synthesis of various types of bio-based polymers [26] and is approved as a plasticizer for rubber items used in the food industry [25]. Furthermore, its sustainability, solubility in methyl methacrylate (MMA) monomer, and relatively high boiling point $\left(313^{\circ} \mathrm{C}\right)$ give it potential as a natural plasticizing agent to develop lowmodulus PMMA bone cement. Castor oil molecules are small enough to arrange themselves between the polymer chains and reduce the modulus by increasing the fluidity of the cured material.

The aim of this study was to evaluate the feasibility of using castor oil as a plasticizer to obtain lowmodulus PMMA bone cements. The mechanical properties of interest were the compressive modulus, and the compressive yield strength $\left(\sigma_{\mathrm{y}}\right)$. The effect of castor oil on the curing properties was assessed in terms of the doughing time $\left(t_{\text {dough }}\right)$, the setting time $\left(t_{\text {setting }}\right)$, the maximum polymerization temperature $\left(T_{\max }\right)$, and the curing profiles, namely, the complex viscosity-versus-time $\left(\eta^{*}-t\right)$ curves. Finally, the cytotoxicity of extracts from the different formulations was tested on cells of the osteoblast-like cell line MG63. The alamarBlue ${ }^{\circledR}$ assay was used to assess the cell viability, whereas the cell morphological changes were investigated by Scanning Electron Microscopy (SEM).

\section{Materials}

All chemicals were purchased from Sigma-Aldrich (Sigma-Aldrich, St. Louis, MO, USA) unless otherwise stated. The commercially available bone cement Osteopal ${ }^{\circledR}$ V (Heraeus Medical GmbH, Hanau, Germany), was used in all experiments. The powder phase is composed of $54.6 \mathrm{wt} \%$ poly(methyl acrylate-co-methyl methacrylate), $45.0 \%$ zirconium dioxide, $0.4 \%$ benzoyl peroxide and an unspecified amount of chlorophyll VIII. The liquid phase is composed of $92 \mathrm{wt} \%$ methyl methacrylate, $2 \mathrm{wt} \%$ $\mathrm{N}, \mathrm{N}$-dimethyl-p-toluidine and $6 \mathrm{wt} \%$ other additives (i.e., chlorophyll VIII and hydroquinone). The liquid-to-powder ratio (without the castor oil) was maintained at $0.38 \mathrm{ml} / \mathrm{g}$ as specified by the fabricant. Castor oil was used without further modification.

\section{Methods}

\subsection{Specimen preparation}

One group of unmodified specimens and three groups of modified specimens were prepared, where the modified specimens contained 2.5, 7.5 or $12 \mathrm{wt} \%$ of castor oil. The specimens were identified as OPV, OPV2.5, OPV7.5 and OPV 12.0, respectively. The modified liquid phases were made by dissolving an appropriate amount of castor oil in the liquid phase by rotary motion using a Vortex-Genie 2 (Scientific Industries, Inc., Bohemia, NY, USA). The specimens were prepared by adding the liquid phase to the powder phase in a glass mortar and mixing with a metal spatula for $30 \mathrm{~s}$. These mixtures were used to prepare specimens for the compression tests, to determine the curing properties, and to perform rheology. The cement preparation for the cytotoxicity tests is described in the cytotoxicity assays section. 


\subsection{Compression tests}

The specimens for the compression tests were made in Teflon ${ }^{\circledR}$ molds and have a size of $6 \mathrm{~mm}$ diameter and $12 \mathrm{~mm}$ height as stipulated in ASTM F451-08 [27] standard. The specimens were stored at $T=21 \pm 1^{\circ} \mathrm{C}$, and tested after $24 \mathrm{~h}$ using an AGS-H universal materials testing machine (Shimadzu, Kyoto, Japan) at a crosshead displacement rate of $25.4 \mathrm{~mm} / \mathrm{min}$. The properties $E$ and $\sigma_{\mathrm{y}}$ were obtained from the load-versus-displacement curves.

\subsection{Curing properties}

The $t_{\text {dough }}, t_{\text {setting }}$ and $T_{\max }$ were determined as described in ASTM F451-08 standard.

\subsection{Rheometry}

The progress of the viscosity during cement curing was assessed in the form of $\eta^{*}-t$ curve. A stresscontrolled rheometer (AR2000; TA instruments, New Castle, DE, USA) equipped with a parallel-plate geometry, was used in a time-sweep step mode at a constant frequency of $5 \mathrm{~Hz}$. The upper titanium plate had a diameter of $19 \mathrm{~mm}$ and the gap was set at $1500 \mu \mathrm{m}$. The displacement amplitude was $5 \times 10^{-4} \mathrm{rad}$ and the temperature of the lower plate was maintained at $23^{\circ} \mathrm{C}$ [28].

\subsection{Cytotoxicity assays}

MG-63 human osteoblast-like cells (HPACC, Salisbury, UK) were expanded in DMEM/F12 culture medium supplemented with 10\% fetal bovine serum (Hyclone, Logan, UT, USA), 2 mM glutamine, $100 \mathrm{U} / \mathrm{ml}$ penicillin and $100 \mu \mathrm{g} / \mathrm{ml}$ streptomycin. Cells were cultured in $75 \mathrm{~cm}^{2}$ flasks and incubated at $37^{\circ} \mathrm{C}$ in a humidified atmosphere with $5 \% \mathrm{CO}_{2}$. At confluence, the cells were detached using trypsin/EDTA. The cells were then plated into 24-well culture-plates at a density of $30,000 \mathrm{cells} / \mathrm{cm}^{2}$ and cultured for $24 \mathrm{~h}$ before starting the cytotoxicity assays.

The cytotoxicity of extracts was assessed according to the ISO-10993 standard [29]. The extracts from five different types of specimens were tested. The specimen preparation was done under sterile conditions. The castor oil was filtered using a $0.45 \mu \mathrm{m}$ syringe-filter prior to use. The first three specimens corresponded to one unmodified cement (OPV) and two modified cements with 7.5 (OPV7.5), and 12.0 wt\% (OPV12.0) castor oil; the other two specimens corresponded to two cements modified with 7.5 (OPV7.5(a)), and $12.0 \mathrm{wt} \%$ (OPV12.0(a)) castor oil, in which the castor oil was added $15 \mathrm{~s}$ after the commencement of mixing. The purpose of this practice was to find possible time and dissolution effects of castor oil when the polymerization had already started. The materials OPV7.5 and OPV12.0 were chosen for the cytotoxicity assays since these exhibited the most prominent property changes respect to OPV. The cement specimens were produced in $50 \mathrm{ml}$ tubes by adding the liquid phase into the powder phase. A cap vibrator (Ivoclar Vivadent AB, Solna, Sweden) was used to mix the two phases during a total of $30 \mathrm{~s}$ in all cases. After $2.5 \mathrm{~min}$ from the commencement of mixing, the culture medium (see above) was added to the tubes at a cement-to-medium ratio of $200 \mathrm{mg} / \mathrm{ml}$. The extraction was done in an incubator over $24 \mathrm{~h}$ at $37^{\circ} \mathrm{C}$. The extraction media were subsequently filtered with a $0.20 \mu \mathrm{m}$ syringefilter and added to the cells. The cells were kept in the media for 1 day and 3 days, respectively. The recovery group corresponded to cells whose medium was replaced by standard medium after 1 day of cultivation. The cell viability was assessed at day 1 and day 3 (including the recovery group) using the alamarBlue ${ }^{\circledR}$ viability assay (Invitrogen Corporation, Carlsbad, CA, USA) according to the instructions from the supplier. 


\subsection{Scanning electron microscopy}

The specimens for SEM were produced by plating, and culturing the cells on Thermanox ${ }^{\mathrm{TM}}$ cover slips as described above. The media were aspirated after 1 and 3 days, the specimens were rinsed twice in Hank's balanced salt solution (HBSS), and the cells were fixed in a solution of $2.5 \%$ glutaraldehyde in HBSS for at least $24 \mathrm{~h}$. The cells were washed again in HBSS and dehydrated in a series of aqueous ethanol solutions $(10,30,50,70,90$ and $100 \%)$ for 10 min each. Desiccation of cells was carried out in hexamethyldisilazane/ethanol solutions $(1: 2,2: 1)$ and pure hexamethyldisilazane for $15 \mathrm{~min}$ each. The specimens were dried in a fume hood and sputter-coated with a $5 \mathrm{~nm}$ layer of palladium prior to SEM investigations. The images were only intended for morphological analysis, and thus taken from areas with similar cell density.

\subsection{Statistical analysis}

Statistical analysis was done in IBM SPSS Statistics 19 (IBM, Chicago, IL, USA) using ANOVA at a significance level of $\alpha=0.05$. Tamhane's post-hoc test was used since equal variance could not be confirmed.

\section{Results}

\subsection{Compression tests}

The results of the compression tests are shown in Fig. 1. The statistical analysis gave $p \leqslant 0.02$ for both $\sigma_{\mathrm{y}}$ and $E$ in all cases, indicating statistically significant differences between OPV and the other groups containing castor oil.

\subsection{Curing properties}

The curing properties are shown in Fig. 2. Tamhane's test for $T_{\max }$ showed statistically significant differences between the groups OPV and OPV7.5, and OPV and OPV12.0 $(p \leqslant 0.01)$. The doughing

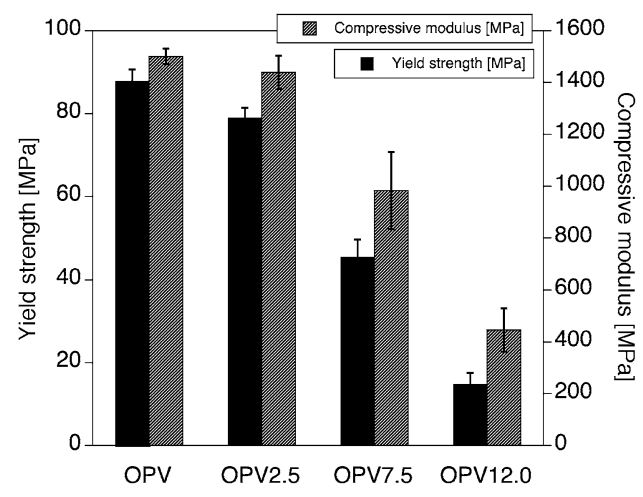

Fig. 1. Yield strength and Young's modulus of PMMA bone cements in compression, prepared with different concentrations of castor oil in the liquid phase. The error bars correspond to the standard deviation $(N=11,15,18$ and 16 , respectively).

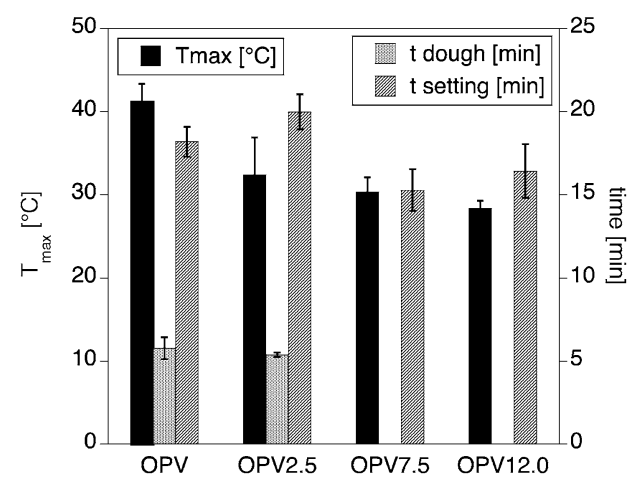

Fig. 2. Curing properties $\left(T_{\max }, t_{\text {dough }}\right.$ and $\left.t_{\text {setting }}\right)$ of PMMA bone cements prepared with different concentrations of castor oil in the liquid phase. The error bars correspond to the standard deviation $(N=5,3,3$ and 3 , respectively). 


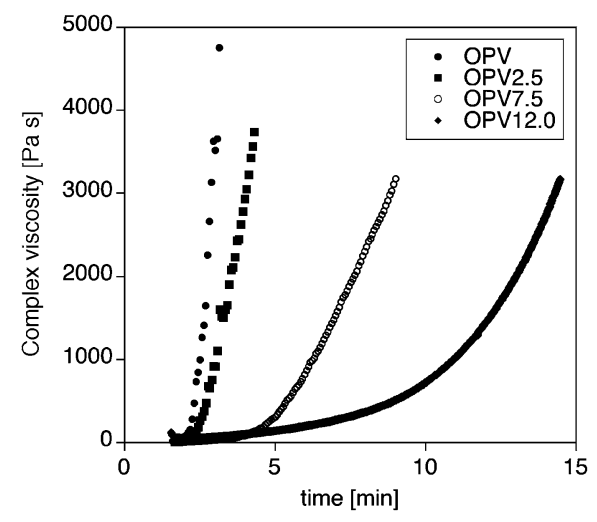

Fig. 3. Typical $\eta^{*}-t$ profiles of PMMA bone cements prepared with different concentrations of castor oil in the liquid phase.

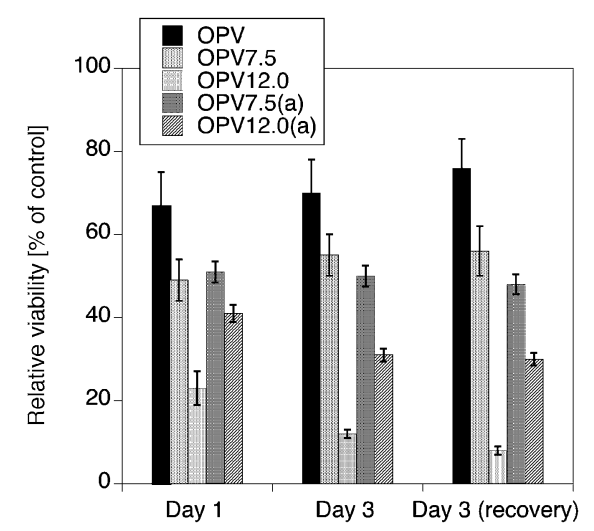

Fig. 4. Viability of MG-63 cells after cultivation in extraction media from the different formulations relative to the viability of cells cultured in standard medium. The error bars correspond to the standard deviation $(N=12)$.

time could not be determined for OPV7.5 and OPV12.0 since no fibers were formed between the glove and the dough. The analysis for $t_{\text {setting }}$ showed no significant differences between the groups $(p \geqslant 0.05)$.

\subsection{Rheometry}

Typical $\eta^{*}-t$ profiles for the different formulations are shown in Fig. 3.

\subsection{Cytotoxicity assays}

The results of the alamarBlue ${ }^{\circledR}$ viability assay are shown in Fig. 4. The statistical analysis revealed the following: (a) increasing the concentration of castor oil significantly $(p \leqslant 0.02)$ lowered the cell viability with respect to OPV in all cases except OPV7.5(a) at day $3(p=0.30)$; (b) for 7.5\% castor oil, there were no significant differences $(p=1.00)$ between pre-dissolving it in the liquid and adding it later; (c) for $12.0 \%$ castor oil in the day 1, there was a significant difference between pre-dissolving it and adding it later $(p=0.01)$, whereas for day 3 and the recovery group, the differences can be considered non-significant $(p \geqslant 0.05)$.

\subsection{Scanning electron microscopy}

The SEM images of the cells at day 1 are shown in Fig. 5.

\section{Discussion}

Some of the current clinical and biomechanical data support the hypothesis that VP with PMMA is the cause of further development of fractures in adjacent vertebrae. One possible explanation to this is the high Young's modulus-disparity between commercially available PMMA bone cements, and the naturally occurring cancellous bone. This difference results in local stiffening, and abnormal load transfer, in particular when maximum filling is used in surgery $[15,16]$. Therefore, low-modulus cements may improve the outcome of VP. In this study we evaluated the use of castor oil as a natural plasticizer for 


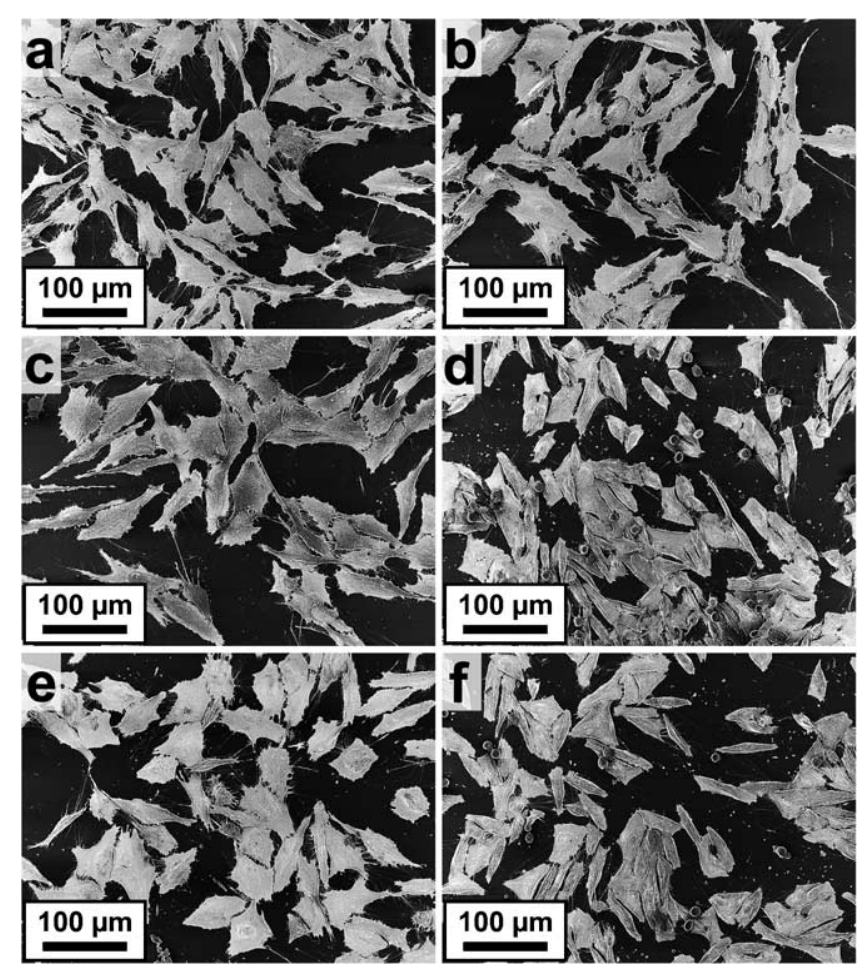

Fig. 5. Scanning electron microscopy micrographs acquired after 1 day of cultivation in a standard culture medium (a); and in culture media with extracts from: OPV (b); OPV7.5 (c); OPV12.0 (d); OPV7.5(a) (e); OPV12.0(a) (f).

PMMA bone cement. PMMA bone cement has two main effects following VP: (a) axial stiffening, and (b) axial strengthening [4], which are related to $E$ and $\sigma_{\mathrm{y}}$ of the cement, respectively. As previously stated, a cause of adjacent vertebral fractures after VP may be the high $E$ of the PMMA bone cement relative to the naturally occurring cancellous bone. The compression tests (Fig. 1) revealed a decrease of $\sigma_{\mathrm{y}}$ from 88 to $15 \mathrm{MPa}$ as well as $E$ from 1500 to $446 \mathrm{MPa}$, when the amount of castor oil was increased from 0 to $12.0 \mathrm{wt} \%$, respectively. Unlike most commercially available bone cements, both of these modified bone cement values fell within the range of cancellous bone.

The curing properties (Fig. 2) of the PMMA bone cements containing castor oil were investigated due to their clinical importance. $T_{\max }$ decreased significantly from 41.3 to $25.6^{\circ} \mathrm{C}$, when increasing the amount of castor oil from 0 to $12.0 \mathrm{wt} \%$. This effect is beneficial since previous reports suggest that temperatures above $50^{\circ} \mathrm{C}$ for more than 1 min might damage the bony tissue [30,31]. $t_{\text {dough }}$ is indicative of the maximum injection or working time. $t_{\text {dough }}$ did not significantly change between OPV, and OPV2.5, which had $t_{\text {dough }}$ values of 5.8, and 5.4 min, respectively. It should be noted that $t_{\text {dough }}$ was not detectable at higher concentrations of castor oil when using the standard method, since the fiber formation was prevented by the oily content of the mixture. $t_{\text {setting }}$ is thus more useful as it is an indication of the time it takes for the bone cement to reach its maximum mechanical strength. $t_{\text {setting }}$ did not significantly change between any of the groups and the values were between 15.2 and 20.0 min. This suggests that the mechanical properties, and the maximum polymerization temperature could be modified towards lower values without altering the setting time of the standard formulation.

Bone cement extravasation has been shown to occur in more than $70 \%$ of the VP procedures [32]. Although this rarely result in complications, there have been reports on paraplegia, spinal cord and 
nerve root compression, pulmonary embolisms and even death [33]. Bone cements with appropriate $\eta^{*}-t$ behavior might have a reduced risk of extravasation. A previous in vitro study has suggested that extravasation risk might be reduced using cements with an initial viscosity of at least $100 \mathrm{~Pa} \cdot \mathrm{s}$ with a maximum of $200 \mathrm{~Pa} \cdot \mathrm{s}$ during the injection time before curing [34]. The present results showed that the time to reach a certain viscosity increased when increasing the amount of castor oil from 0 to $12.0 \mathrm{wt} \%$, thereby increasing the working time. The shape of the viscosity curves also depended on the castor oil concentration. Figure 3 shows how OPV exhibited a rapid viscosity increase starting at $2 \mathrm{~min}$, and reached $4744 \mathrm{~Pa} \cdot \mathrm{s}$ after $3.2 \mathrm{~min}$ from the commencement of the mixing. In contrast, OPV12.0 remained below $200 \mathrm{~Pa} \cdot \mathrm{s}$ up to $6 \mathrm{~min}$ and reached $3768 \mathrm{~Pa} \cdot \mathrm{s}$ after $14.5 \mathrm{~min}$. This indicates that the addition of up to $12.0 \mathrm{wt} \%$ castor oil is useful to increase the time that the cement remains below a relatively low $\eta^{*}$ without affecting the $t_{\text {setting }}$.

The cytotoxic effects of the extracts from the different PMMA cement formulations on MG-63 human osteoblast-like cells were assessed using the alamarBlue ${ }^{\circledR}$ cell viability assay (Fig. 4), and SEM micrographs (Fig. 5). The use of all extraction media resulted in a lower viability compared to the control group. The extraction media from unmodified PMMA induced the lowest cytotoxic effect, with approximately $30 \%$ higher viability. Additionally, the viability decreased when the amount of castor oil increased. Changing the media after 1 day did not significantly improve the viability (recovery group). Thus, after an initial viability decrease due to cell death, the cell proliferation in the OPV, OPV7.5 and OPV7.5(a) groups was comparable to that of the control, and resulted in constant viability values along the 3 days. This may be related to evaporation of toxic substances from the media, which in turn supports a normal cell proliferation. In contrast, there was a trend to lower cell viability for both OPV12.0 and OPV12.0(a) after 3 days. This was related to a higher initial cytotoxic effect, which caused irreversible damage to the cells and reduced their proliferation. There were no significant differences in cell viability between OPV7.5 and OPV7.5(a). In contrast, the cell viability of the OPV12.0 group was significantly lower than when the castor oil was added after $15 \mathrm{~s}$ from the commencement of the mixing (OPV12.0(a)). Accordingly, it appears that it is not the presence of castor oil itself that affects the cell viability, but rather is its interference with the chemical reaction between the liquid and the powder phase.

The cell confluency corresponded to the data obtained by the alamarBlue ${ }^{\circledR}$ assay, as seen from initial light microscopy (images not shown). However, some of the extraction media also affected the morphology of the MG-63 cells. SEM showed no appreciable difference in cell morphology between the control (standard culture medium) group (Fig. 5a), and the OPV group (Fig. 5b). In both cases, the cells exhibited a fibroblast-like morphology with numerous filopodia. On the other hand, the cells corresponding to the modified formulations (Fig. 5c-f) were less fibroblastic, smaller, and exhibited a cuboidal morphology. These features were less noticeable for the formulations with $7.5 \mathrm{wt} \%$ castor oil (Fig. $5 \mathrm{c}, \mathrm{e}$ ), and more pronounced for $12 \mathrm{wt} \%$ castor oil (Fig. 5d, f). The morphology of the cells did not remarkably change until day 3 (images not shown). The same was observed for the specimens of the recovery groups. Regardless, a full confluent cell monolayer was found in all specimens except for cells cultured in extracts of OPV12 and OPV12(a). The morphological change and the cell viability data indicated that the cells were irreversibly damaged during the initial contact with the material extracts of the $12.0 \%$ castor oil-containing formulations.

These cytotoxicity results suggest an increased presence of one or more toxic components in the media that are affecting cell adhesion, functionality, or structure of the cytoskeleton. The longer working time of the castor oil-modified cements may expose the cells to the monomer for longer time periods in vivo. Moreover, the castor oil might prevent polymerization of the cement to a certain extent by inhibiting 
one of the chemical reactions involved. For instance the phenyl radicals from the reaction between benzoyl peroxide and $\mathrm{N}, \mathrm{N}$-dimethyl- $p$-toluidine, could attack the double bonds in the unsaturated fatty acyl groups of castor oil. The number of these double bonds increases when increasing the concentration of castor oil, thereby consuming more radicals and leaving behind more unreacted monomer. Accordingly, increased exposure to unreacted monomer might be the cause of cell shrinkage and lower viability in the presence of castor oil. However, it should be noticed that this is a worst-case test in which the cell media are directly exposed to the materials during curing under steady conditions. The negative effects are likely to be less in vivo due to fluid buffering and a higher cell number.

\section{Conclusions}

The addition of up to $12 \mathrm{wt} \%$ castor oil significantly decreases $\sigma_{\mathrm{y}}$ from 88 to $15 \mathrm{MPa}$, and $E$ from 1500 to $446 \mathrm{MPa}$. These properties depend on the amount of castor oil and a Young's modulus closer to that of osteoporotic vertebrae was obtained. The results also show that up to $12 \mathrm{wt} \%$ castor oil decreases $T_{\max }$ from 41.3 to $25.6^{\circ} \mathrm{C}$, without significantly affecting the $t_{\text {setting, }}$, which would allow for a milder polymerization environment. It is also found that up to $12.0 \mathrm{wt} \%$ castor oil modifies the viscosity of the commercial fast-setting formulation to increase its working time. However, the addition of castor oil has a negative effect on viability and morphology of osteoblastic MG-63 cells, which is considered to be due to an increased presence of unreacted methyl methacrylate monomer. Further investigations are necessary to improve the cell viability of castor oil-modified bone cements for use in vertebroplasty.

\section{Acknowledgements}

Funding from the European Union for The Osteoporotic Virtual Physiological Human project (VPHOP FP7-ICT2008-223865) and the SPINEGO project (FP7-ICT2008-223865), as well as VINNOVA (VINNMER 2010-02073) is gratefully acknowledged.

\section{References}

[1] G. Lewis, Injectable bone cements for use in vertebroplasty and kyphoplasty: State-of-the-art review, Journal of Biomedical Materials Research Part B: Applied Biomaterials 76B (2006), 456-468.

[2] M.A. Liebschner, W.S. Rosenberg and T.M. Keaveny, Effects of bone cement volume and distribution on vertebral stiffness after vertebroplasty, Spine 26 (2001), 1547-1554.

[3] P.F. Heini, U. Berlemann, M. Kaufmann, K. Lippuner, C. Fankhauser and P. van Landuyt, Augmentation of mechanical properties in osteoporotic vertebral bones - a biomechanical investigation of vertebroplasty efficacy with different bone cements, European Spine Journal 10 (2001), 164-171.

[4] G. Baroud, J. Nemes, S.J. Ferguson and T. Steffen, Material changes in osteoporotic human cancellous bone following infiltration with acrylic bone cement for a vertebral cement augmentation, Computer Methods in Biomechanics and Biomedical Engineering 6 (2003), 133-139.

[5] L. Hernandez, M.E. Muñoz, I. Goñi and M. Gurruchaga, New injectable and radiopaque antibiotic loaded acrylic bone cements, Journal of Biomedical Materials Research Part B: Applied Biomaterials 87B (2008), 312-320.

[6] S.M. Kurtz, M.L. Villarraga, K. Zhao and A.A. Edidin, Static and fatigue mechanical behavior of bone cement with elevated barium sulfate content for treatment of vertebral compression fractures, Biomaterials 26 (2005), 3699-3712.

[7] A. Boger, M. Bohner, P. Heini, S. Verrier and E. Schneider, Properties of an injectable low modulus PMMA bone cement for osteoporotic bone, Journal of Biomedical Materials Research Part B: Applied Biomaterials 86B (2008), 474-482.

[8] A. Nazarian, D. von Stechow, D. Zurakowski, R. Müller and B. Snyder, Bone volume fraction explains the variation in strength and stiffness of cancellous bone affected by metastatic cancer and osteoporosis, Calcified Tissue International $\mathbf{8 3}$ (2008), 368-379. 
[9] F. Grados, C. Depriester, G. Cayrolle, N. Hardy, H. Deramond and P. Fardellone, Long-term observations of vertebral osteoporotic fractures treated by percutaneous vertebroplasty, SAS Journal 3 (2009), 118-122.

[10] A.A. Uppin, J.A. Hirsch, L.V. Centenera, B.A. Pfiefer, A.G. Pazianos and I.S. Choi, Occurrence of new vertebral body fracture after percutaneous vertebroplasty in patients with osteoporosis, Radiology 226 (2003), 119-124.

[11] A.T. Trout, D.F. Kallmes and T.J. Kaufmann, New fractures after vertebroplasty: adjacent fractures occur significantly sooner, AJNR Am. J. Neuroradiol. 27 (2006), 217-223.

[12] W.-J. Chen, Y.-H. Kao, S.-C. Yang, S.-W. Yu, Y.-K. Tu and K.-C. Chung, Impact of cement leakage into disks on the development of adjacent vertebral compression fractures, Journal of Spinal Disorders \& Techniques 23 (2010), 35-39.

[13] G. Baroud, P. Heini, J. Nemes, M. Bohner, S. Ferguson and T. Steffen, Biomechanical explanation of adjacent fractures following vertebroplasty [letter], Radiology 229 (2003), 606-608.

[14] G. Baroud, J. Nemes, P. Heini and T. Steffen, Load shift of the intervertebral disc after a vertebroplasty: a finite-element study, European Spine Journal 12 (2003), 421-426.

[15] U. Berlemann, S.J. Ferguson, L.-P. Nolte and P.F. Heini, Adjacent vertebral failure after vertebroplasty: a biomechanical investigation, J. Bone Joint Surg. Br. 84-B (2002), 748-752.

[16] R. Wilcox, The biomechanical effect of vertebroplasty on the adjacent vertebral body: a finite element study, Proceedings of the Institution of Mechanical Engineers, Part H: Journal of Engineering in Medicine 220 (2006), 565-572.

[17] C.A.H. Klazen, A. Venmans, J. De Vries et al., Percutaneous vertebroplasty is not a risk factor for new osteoporotic compression fractures: results from VERTOS II, American Journal of Neuroradiology 31 (2010), 1447-1450.

[18] A. Boger, P. Heini, M. Windolf and E. Schneider, Adjacent vertebral failure after vertebroplasty: a biomechanical study of low-modulus PMMA cement, European Spine Journal 16 (2007), 2118-2125.

[19] A. Boger, A. Bisig, M. Bohner, P. Heini and E. Schneider, Variation of the mechanical properties of PMMA to suit osteoporotic cancellous bone, Journal of Biomaterials Science, Polymer Edition 19 (2008), 1125-1142.

[20] S. Beck and A. Boger, Evaluation of the particle release of porous PMMA cements during curing, Acta Biomater. 5 (2009), $2503-2507$.

[21] W.M. Lam, H.B. Pan, M.K. Fong et al., In vitro characterization of low modulus linoleic acid coated strontium-substituted hydroxyapatite containing PMMA bone cement, Journal of Biomedical Materials Research Part B: Applied Biomaterials 96B (2010), 76-83.

[22] D. Martinz and J. Quadros, Compounding PVC with renewable materials, Plastics, Rubber and Composites 37 (2008), 459-464.

[23] T.R. Kukreja, R.C. Chauhan, S. Choe and P.P. Kundu, Effect of the doses and nature of vegetable oil on carbon black/rubber interactions: Studies on castor oil and other vegetable oils, Journal of Applied Polymer Science 87 (2003), $1574-1578$.

[24] P. Raju, V. Nandanan and S.K.N. Kutty, A study on the use of castor oil as plasticizer in natura rubber compounds, Progress in Rubber, Plastics and Recycling Technology 23 (2007), 169-180.

[25] Final report on the safety assessment of Ricinus communis (castor) seed oil, hydrogenated castor oil, glyceryl ricinoleate, glyceryl ricinoleate se, ricinoleic acid, potassium ricinoleate, sodium ricinoleate, zinc ricinoleate, cetyl ricinoleate, ethyl ricinoleate, glycol ricinoleate, isopropyl ricinoleate, methyl ricinoleate, and octyldodecyl ricinoleate, International Journal of Toxicology 26 (2007), 31-77.

[26] V. Sharma and P.P. Kundu, Addition polymers from natural oils - a review, Progress in Polymer Science 31 (2006), 983-1008.

[27] ASTM F451-08, ASTM International, West Conshohocken, PA, USA, 2008.

[28] D.F. Farrar and J. Rose, Rheological properties of PMMA bone cements during curing, Biomaterials 22 (2001), 30053013.

[29] ISO-10993, ISO, 2001.

[30] R.A. Eriksson and T. Albrektsson, The effect of heat on bone regeneration: An experimental study in the rabbit using the bone growth chamber, Journal of Oral and Maxillofacial Surgery 42 (1984), 705-711.

[31] R.A. Eriksson, T. Albrektsson and B. Magnusson, Assessment of bone viability after heat trauma: a histological, histochemical and vital microscopic study in the rabbit, Journal of Plastic and Reconstructive Surgery and Hand Surgery 18 (1984), 261-268.

[32] R. Schmidt, B. Cakir, T. Mattes, M. Wegener, W. Puhl and M. Richter, Cement leakage during vertebroplasty: an underestimated problem?, European Spine Journal 14 (2005), 466-473.

[33] R. Lador, N. Dreiangel, P.J. Ben-Galim and J.A. Hipp, A pictorial classification atlas of cement extravasation with vertebral augmentation, The Spine Journal 10 (2010), 1118-1127.

[34] M. Loeffel, S.J. Ferguson, L.-P. Nolte and J.H. Kowal, Vertebroplasty: experimental characterization of polymethylmethacrylate bone cement spreading as a function of viscosity, bone porosity, and flow rate, Spine 33 (2008), 1352-1359. 